\title{
ON THE CLOSURE OF THE NUMERICAL RANGE OF AN OPERATOR
}

\section{S. K. BERBERIAN AND G. H. ORLAND}

If $T$ is a bounded linear mapping (briefly, operator) in a Hilbert space $\mathfrak{H C}$, the numerical range of $T$ is the set $W(T)=\{(T x, x):\|x\|$ $=1\}$; thus $W(T)$ is convex $[8$, p. 131], and its closure $\mathrm{cl}[W(T)]$ is compact and convex. Roughly speaking, in this note we observe that $\operatorname{cl}[W(T)]$ can be uniquely defined for an element $T$ of an abstract $C^{*}$-algebra, while $W(T)$ cannot. The $C^{*}$-algebra setting yields an extension of the spectral convexity theorem [8, p. 327] to nonnormal operators (Corollary 1 of Theorem 3), as well as a reformulation of a theorem of C. Berger (Corollary 2 of Theorem 3).

1. An algebraic characterization of the closure of the numerical range. Let $T$ be an operator in a Hilbert space 3 . Our objective in this section is to describe $\mathrm{cl}[W(T)]$ in algebraic terms; since $\mathrm{cl}[W(T)]$ is the intersection of all closed half planes $H$ containing it, it is sufficient to describe those $H$ that contain $W(T)$ (see Theorem 1). We write $\operatorname{Re} \lambda$ for the real part of a complex number $\lambda$; analogously we define $\operatorname{Re} T=\frac{1}{2}\left(T^{*}+T\right)$.

Lemma 1 . $\operatorname{Re} T \geqq 0$ if and only if $(T-\alpha I)^{*}(T-\alpha I) \geqq \alpha^{2} I$ for all $\alpha<0$.

Proof. For any real number $\alpha$,

$$
(T-\alpha I)^{*}(T-\alpha I)-\alpha^{2} I=T^{*} T-\alpha\left(T^{*}+T\right) .
$$

If the left side of this equation is $\geqq 0$ for all $\alpha<0$, then for all $\alpha<0$ we have $\alpha\left(T^{*}+T\right) \leqq T^{*} T, T^{*}+T \geqq\left(T^{*} T\right) / \alpha$, and $T^{*}+T \geqq 0$ results on letting $\alpha \rightarrow-\infty$; thus $\operatorname{Re} T \geqq 0$. If, conversely, $\operatorname{Re} T \geqq 0$, then for any $\alpha<0$ we have $T^{*}+T \geqq 0 \geqq\left(T^{*} T\right) / \alpha$, and the steps of the above argument may be reversed.

LEMma 2. Let $H_{0}=\{\lambda: \operatorname{Re} \lambda \geqq 0\}$. Then $W(T) \subset H_{0}$ if and only if $(T-\lambda I)^{*}(T-\lambda I) \geqq(\operatorname{Re} \lambda)^{2} I$ for all $\lambda$ not in $H_{0}$.

Proof. Since $((\operatorname{Re} T) x, x)=\operatorname{Re}(T x, x)$, clearly $W(T) \subset H_{0}$ if and only if $\operatorname{Re} T \geqq 0$. If $\lambda=\alpha+i \beta$ with $\alpha$ and $\beta$ real, then $T-\lambda I=(T-i \beta I)$ $-\alpha I$, and $\operatorname{Re} T=\operatorname{Re}(T-i \beta I)$. Fixing any real $\beta$, by Lemma 1 we have $(T-\lambda I)^{*}(T-\lambda I) \geqq \alpha^{2} I$ for all $\alpha<0$ if and only if $\operatorname{Re}(T-i \beta I) \geqq 0$, that is, $\operatorname{Re} T \geqq 0$.

If $H$ is a set of complex numbers, we write

Received by the editors June 7, 1966. 


$$
\operatorname{dist}(\lambda, H)=\inf \{|\lambda-\mu|: \mu \in H\} .
$$

TheOREM 1. Let $H$ be a closed half plane of the complex plane. Then $W(T) \subset H$ if and only if $(T-\lambda I)^{*}(T-\lambda I) \geqq[\operatorname{dist}(\lambda, H)]^{2} I$ for all complex numbers $\lambda$.

Proof. Obviously we need consider only $\lambda$ not in $H$. The case $H=H_{0}$ is covered by Lemma 2. In general, we have $f(H)=H_{0}$ for a suitable linear function $f(\lambda) \equiv \mu \lambda+\tau$ with $|\mu|=1$. Let $S=f(T)$ $=\mu T+\tau I$. Evidently $W(S)=f[W(T)]$, thus $W(T) \subset H$ if and only if $W(S) \subset H_{0}$. Also $S-f(\lambda) I=\mu(T-\lambda I)$, hence $(S-f(\lambda) I)^{*}(S-f(\lambda) I)$ $=(T-\lambda I)^{*}(T-\lambda I)$. Finally, $\operatorname{dist}\left(f(\lambda), H_{0}\right)=\operatorname{dist}(f(\lambda), f(H))$ $=\operatorname{dist}(\lambda, H)$. Thus the general case follows on applying the special case $H=H_{0}$ to the operator $S$.

Writing $\sigma(T)$ for the spectrum of $T$, we may reformulate Theorem 1 in terms of resolvents:

CoRollary. Let $H$ be a closed half plane of the complex plane. Then $W(T) \subset H$ if and only if

(i) $\sigma(T) \subset H$, and

(ii) $\left\|(T-\lambda I)^{-1}\right\| \leqq 1 / \operatorname{dist}(\lambda, H)$ for all $\lambda$ not in $H$.

Proof. If $W(T) \subset H$, then $\sigma(T) \subset \operatorname{cl}[W(T)] \subset H$ (cf. [2, Lemma 1]), and so (i) holds. On the other hand if $\lambda$ is not in $\sigma(T)$ or in $H$, the inequality in (ii) is equivalent to

$$
\operatorname{dist}(\lambda, B)\left\|(T-\lambda I)^{-1} y\right\| \leqq\|y\|
$$

for all vectors $y$, that is,

$$
\operatorname{dist}(\lambda, H)\|x\| \leqq\|(T-\lambda I) x\|
$$

for all vectors $x$, that is,

$$
(T-\lambda I)^{*}(T-\lambda I) \geqq[\operatorname{dist}(\lambda, H)]^{2} I .
$$

The corollary now follows at once from Theorem 1 .

2. $C^{*}$-algebras. Let $A$ be a $C^{*}$-algebra with unity $1[4$, p. 6]. By the Gel'fand-Naimark theorem there exists a faithful *-representation $a \rightarrow T_{a}$ of $A$ as operators on a suitable Hilbert space, with $T_{\mathbf{1}}=I$ [4, p. $39 ; 6$, p. 244]. If $a \in A$, we define the closed numerical range of $a$, denoted $\bar{W}(a)$, to be the set $\operatorname{cl}\left[W\left(T_{a}\right)\right]$; the definition is justified by the following theorem:

THEOREM 2. Let $A$ be a $C^{*}$-algebra with unity, and suppose we are given faithful *-representations $a \rightarrow T_{a}$ and $a \rightarrow S_{a}$, as operators on Hilbert spaces $\mathfrak{K C}$ and $\mathfrak{K}$, respectively, such that $T_{1}=I$ and $S_{1}=I$. Then $\mathrm{cl}\left[W\left(T_{a}\right)\right]$ $=\mathrm{cl}\left[W\left(S_{a}\right)\right]$ for all $a$ in $A$. 
Proof. The systems $\left\{T_{a}: a \in A\right\}$ and $\left\{S_{a}: a \in A\right\}$ are $C^{*}$-algebras of operators [4, p. 16], and $T_{a} \rightarrow S_{a}$ is a $*$-isomorphism of one on the other; in particular, $T_{a} \rightarrow S_{a}$ preserves positivity [4, pp. 8 and 12]; [7, $\$ \$ 104$ and 118]. Fix $a$ in $A$. If $H$ is a closed half plane, it is clear from Theorem 1 that $\operatorname{cl}\left[W\left(T_{a}\right)\right] \subset H$ if and only if $\operatorname{cl}\left[W\left(S_{a}\right)\right] \subset H$. Since a closed convex set is the intersection of the closed half planes that contain it, the theorem is proved.

Let us write $\Sigma$ for the set of all normalized states of $A$, that is, the set of all linear forms $f$ on $A$ such that $f(1)=1$ and $f\left(a^{*} a\right) \geqq 0$ for all $a$ in $A$; then $\Sigma$ is a convex subset of the dual space of $A$, and is compact in the weak* topology (cf. [4, p. 37]; [6, p. 222, Lemma 4.6.2]). For any $a$ in $A$ we write $\Sigma(a)=\{f(a): f \in \Sigma\}$. Since the mapping $f \rightarrow f(a)$ is linear and weak* continuous, $\Sigma(a)$ is compact and convex; indeed, we have the following intrinsic characterization of $\bar{W}(a)$ :

Theorem 3. If $A$ is a $C^{*}$-algebra with unity, and $\Sigma$ is the set of all normalized states of $A$, then

$$
\Sigma(a)=\bar{W}(a)
$$

for every $a$ in $A$.

Proof. Each $f$ in $\Sigma$ leads to a canonical *-representation of $A$ on a Hilbert space $\mathscr{K}_{f}$, and by the Gel'fand-Naimark theorem the direct sum of these representations is a faithful *-representation $a \rightarrow T_{a}$ of $A$ as operators on the direct sum $\mathscr{K}$ of the Hilbert spaces $\varkappa_{f}[4$, p. 39]; [6, p. 197]. Fix $a$ in $A$. It is obvious from the construction that $f(a)$ $\in W\left(T_{a}\right)$ for each $f$ in $\Sigma$, thus $\Sigma(a) \subset W\left(T_{a}\right)$. On the other hand if $x \in \mathcal{K},\|x\|=1$, then the formula $f(b)=\left(T_{b} x, x\right)$ defines a normalized state of $A$, and $\left(T_{a} x, x\right)=f(a) \in \Sigma(a)$; varying $x$ we have $W\left(T_{a}\right) \subset \Sigma(a)$, and therefore $\Sigma(a)=W\left(T_{a}\right)$. In particular, for each $a$ in $A, W\left(T_{a}\right)$ is closed and therefore coincides with $\bar{W}(a)$. (This precludes an invariant definition of "numerical range" in the $C^{*}$-algebra setting; the numerical range can be closed in one representation and not closed in another.)

Denote the set of extremal points of $\Sigma$ by $\Sigma_{\theta}$; these are precisely the normalized pure states of $A$, that is, the elements of $\Sigma$ for which the canonical *-representation of $A$ is irreducible [4, p. 37]; [6, p. 223]. For any $a$ in $A$ we write $\Sigma_{e}(a)=\left\{f(a): f \in \Sigma_{e}\right\}$. By the Kreln-Mil'man theorem, $\Sigma$ is the weak* closure of the convex hull of $\Sigma_{\varepsilon}$; it follows readily that for each $a$ in $A, \Sigma(a)$ is the closure of the convex hull of $\Sigma_{e}(a)$. Citing formula (1) we have

Corollary 1. If $A$ is a $C^{*}$-algebra with unity, and $\Sigma_{e}$ is the set of all normalized pure states of $A$, then 


$$
\operatorname{cl}\left[\operatorname{conv} \Sigma_{e}(a)\right]=\bar{W}(a)
$$

for every $a$ in $A$.

If, in particular, $A$ is commutative (and so every $a$ in $A$ is normal), then $\Sigma_{e}$ coincides with the set of characters of $A[4$, p. 23, B3]; [6, p. 229] and one has $\Sigma_{e}(a)=\sigma(a)$ for all $a$ in $A$, where $\sigma(a)$ denotes the spectrum of $a$. Since the convex hull of $\sigma(a)$ is closed (cf. [2]) formula (2) yields

$$
\operatorname{conv} \sigma(a)=\bar{W}(a)
$$

for all $a$ in $A$; thus formula (2) may be viewed as a noncommutative extension of the classical spectral convexity theorem for normal operators $[8$, p. 327]; [2].

Corollary 2. With notations as in Theorem 3, define $\omega(a)$ $=\sup \{|f(a)|: f \in \Sigma\}$. Then $\omega\left(a^{n}\right) \leqq(\omega(a))^{n}$ for all positive integers $n$.

Proof. If $T$ is any operator, write $\omega(T)=\sup \{|\lambda|: \lambda \in W(T)\}$. By a theorem of C. Berger [3], $\omega\left(T^{n}\right) \leqq(\omega(T))^{n}$ for all $n$, and the corollary follows at once from Theorem 3. In particular, if $a$ is in the polar set of $\Sigma$ (that is, if $\omega(a) \leqq 1$ ), then so is $a^{n}$; Berger's result is thus seen to be equivalent to the assertion that the polar set of $\Sigma$ is closed under the formation of powers.

Corollary 3. If $\mathfrak{H}$ is a Hilbert space and $f$ is any normalized state on the $C^{*}$-algebra $\mathfrak{L}(\mathfrak{H C})$ of all operators in $\mathfrak{H}$, then for each $T$ in $\mathfrak{L}(\mathfrak{H C})$ there exists a sequence (depending on $T$ ) of unit vectors $x_{n}$ in KC such that $f(T)=\lim \left(T x_{n}, x_{n}\right)$.

Proof. Applying Theorem 3 to the $C^{*}$-algebra $A=\mathfrak{L}(\mathfrak{H C})$, we have $f(T) \in \bar{W}(T)$ by formula (1); but $\bar{W}(T)=\mathrm{cl}[W(T)]$ since the identity representation may be employed in the definition of $\bar{W}(T)$. We remark that the proof of Theorem 3 yields a canonical faithful $*$-representation $T \rightarrow T^{\sharp}$ of $\mathscr{L}(\mathcal{K})$ on a suitable Hilbert space $\mathscr{K}$, in such a way that $W\left(T^{*}\right)$ is closed for every $T$ in $\mathscr{L}(\mathcal{F})$, indeed, $W\left(T^{*}\right)=\mathrm{cl}[W(T)]$. We now present a highly noncanonical representation of this sort.

3. Approximate proper vectors. With the notations of [1], let $\mathfrak{F}$ be a Hilbert space, $\mathscr{K}$ the extension of $\mathfrak{H}$ constructed from a generalized limit, and $T \rightarrow T^{\circ}$ the resulting faithful *-representation of $\mathscr{L}(\mathfrak{F})$ on $K$.

Proposition. For any operator $T$ in $\mathfrak{H}, W\left(T^{\circ}\right)$ is closed; indeed, $W\left(T^{\circ}\right)=\operatorname{cl}[W(T)]$. 
Proof. We recall from [1] the elementary observations that $I^{\circ}=I$, and $T^{\circ} \geqq 0$ if and only if $T \geqq 0$. It follows that $\operatorname{cl}\left[W\left(T^{\circ}\right)\right]=\operatorname{cl}[W(T)]$ (see the proof of Theorem 2). We conclude the proof by showing that $\operatorname{cl}[W(T)] \subset W\left(T^{\circ}\right)$. Suppose $\lambda=\lim \left(T x_{n}, x_{n}\right)$, where $x_{n}$ is a sequence of unit vectors in $\mathcal{H}$. Writing $u=\left\{x_{n}\right\}^{\prime}$ as in [1], we have $\|u\|=1$ and $\left(T^{\circ} u, u\right)=\operatorname{glim}\left(T x_{n}, x_{n}\right)=\lim \left(T x_{n}, x_{n}\right)=\lambda$.

ADDED IN PROOF. E. Berkson has pointed out to us that Theorem 3 is implicit in an article of G. Lumer [Trans. Amer. Math. Soc. 100 (1961), 29-43, Theorem 11] since, on a $C^{*}$-algebra with unity, a continuous linear form $f$ is positive if and only if $f(1)=\|f\|[$ H. F. Bohnenblust and S. Karlin, Ann. of Math. 62 (1955), 217-229, Theorem 10] (see also p. 25 of [1]). Theorem 3 is also covered by Theorem 12 of Bohnenblust and Karlin [loc. cit.]. An elementary proof of Berger's result has been published by C. Pearcy [Michigan Math. J. 13 (1966), 289-291]. .

\section{REFERENCES}

1. S. K. Berberian, Approximate proper vectors, Proc. Amer. Math. Soc. 13 (1962), 111-114.

2. - The numerical range of a normal operator, Duke Math. J. 31 (1964), 479-483.

3. C. Berger, $A$ strange dilation theorem, Abstract 625-152, Notices Amer. Math. Soc. 12 (1965), 590. 1964.

4. J. Dixmier, Les $C^{*}$-algèbres et leurs représentations, Gauthier-Villars, Paris,

5. P. R. Halmos, Introduction to Hilbert space and the theory of spectral multiplicity, Chelsea, New York, 1951.

6. C. E. Rickart, General theory of Banach algebras, Van Nostrand, Princeton, N. J., 1960.

7. F. Riesz and B. Sz.-Nagy, Lȩ̧ons d'analyse fonctionelle, Acad. Sci. Hungary, Akadémiai Kiadó, Budapest, 1952.

8. M. H. Stone, Linear transformations in Hilbert space and their applications to analysis, Colloq. Publ., Vol. 15, Amer. Math. Soc., Providence, R. I., 1932.

The University of Iowa AND

WESLEYAN UNIVERSITY 